\title{
New Adaptive Routing Proposal for DTN
}

\author{
Mamoun Hussein Mamoun \\ Faculty of Computer Science and Information, Al Jouf University \\ Al Jouf, Saudi Arabia
}

\begin{abstract}
In Disruption Tolerant Networks, packet forwarding scheme has a major drawback in terms of network congestion. In order to solve this problem, a New Adaptive Routing Proposal (NARP) has been proposed NARP uses the message forwarding probability scheme in order to increase the delivery ratio and bandwidth utilization. Also, NARP arranges the dropping sequence based on their assigned priority. The priority is determined by the average hop count and average latency. Meanwhile NARP has an ACK list exchange mechanism that is used to purge the redundant messages. Simulation of NARP was carried out and its performance was compared to well known DTN routing protocols: Epidemic Routing, and Spray and Wait Routing. Simulation results show that NARP outperforms them in terms of packet delivery ratio, average latency, and overhead ratio.
\end{abstract}

\section{General Terms}

DTN routing protocols, Opportunistic networks, Adaptive forward ring mechanism, packet dropping policy

\section{Keywords}

DTN routing, New routing mechanism, adaptive forwarding policy, packet dropping policy.

\section{INTRODUCTION}

Delay and Disruption Tolerant Networks are wireless networks where disconnections may occur frequently due to node mobility, limited radio range and power outages. Message delivery delays may be unpredictable since there may never be a complete end-to-end route, or such a route may break or change soon after it has been setup. Typical DTNs are tactical networks [1], vehicular networks [2], interplanetary networks [3] , and nomadic communities networks [4] etc. Different from typical routing procedure in MANET, DTN uses Store-carry-and-forward protocols, such as the Epidemic Routing [5]. In DTN routing protocols, messages are usually replicated among nodes even if most of them have already buffered their copies. These unnecessary copies not only occupy large buffer space but also waste precious wireless bandwidth. Therefore, it is necessary mechanisms to reduce these redundancy forwarding and efficiently arrange the message for transmission..

This paper presents a new protocol for routing in DTN. NARP forwards messages according to an adaptive probability scheme for reducing to increase the delivery ratio and bandwidth utilization. Furthermore, NARP assigns the priority, as a function of a message average latency and average hop count to each message (Dropping Policy). In order to decrease the amount of message copy and make good use of node buffer, a message acknowledgement scheme is also deployed in the routing procedure.

The remainders of this paper are as follows. Section 2 discusses related works on DTN routing. In Section 3, we will describe the proposed routing protocol. Simulations and results, comparing NARP to other well known DTN routing protocols, are presented in Section 4. Finally, Section 5 is the conclusion remark.

\section{RELATED WORK}

In order to decrease the overhead of Epidemic Routing, some improved methods are proposed, including probabilistic forwarding, controlled reproduction named Spray and Wait [6], and single copy routing [7]. The key ideas of these routing algorithms are to predict the node mobility and message distribution, then choose the appropriate next hop according to the statistics of contact history $[8,9]$ or the network topology [10]. Other aspects of DTN routing, such as ferrying, global scheduling and node position are investigated in $[11,12]$.

Different from the above routing solutions, our work is unique in a number of ways. Unlike many existing protocols, NARP does not rely upon the statistic estimate in forwarding process. Furthermore, it introduces the acknowledgement scheme to ensure delivery of message ratio while keep the amount of copies at a low value. Moreover, it deploys multiple metrics to weigh messages for transmission priority and buffer use.

In the acknowledgement scheme: Since messages are forwarded by asynchronous transmission mode in DTN, most of the intermediate nodes of a message can hardly know whether a copy of this message has been delivered to the destination. In this case, even if a message has been successfully delivered, there still exist multiple copies of the message in the network consuming the resources unnecessarily. Therefore, we adopt an acknowledgement vector exchange mechanism that is similar to the analysis in [13] to reduce such overhead.

\section{THE PROPOSED ROUTI NG}

NARP assigns each message with apriority for dropping. NARP uses the average latency and average hop count of a message to calculate this priority. Thus, when the network becomes congested, we consider dropping message with more hops and larger latency first. This is the idea of the buffer management policy. But this policy usually cannot observably reduce the network overhead, so NARP uses the message forwarding probability scheme for reducing to increase the delivery ratio and bandwidth utilization. The message forwarding scheme, the dropping policy, and transmission priority policy form the NARP strategy.

\subsection{Forwarding Probability Scheme}

In NARP, each mobile node employs a neighbor discovery algorithm to create and maintain a bidirectional link with a newly discovered node, using the HELLO mechanism. NARP uses the summary vector exchange mechanism to reduce the transmission. Using this mechanism, a pair of mobile nodes exchange their message lists, and only transmit messages which are not found in each other's message lists. 
The forwarding probability for each message buffered in a mobile node id given by [14]

$$
P_{f}=\left\{\begin{array}{cl}
P_{\text {init }} & R D_{i}\left(T_{s}\right) \leq p_{t h} \\
P_{\text {init }}\left(\frac{1-R D_{i}\left(T_{s}\right)}{R D_{i}\left(T_{s}\right)}\right) & R D_{i}\left(T_{s}\right) \succ p_{t h}
\end{array}\right.
$$

Where $P_{\text {init }}, p_{\text {th }}$ are predefined constants and $R D_{i}\left(T_{s}\right)$ is the replication density for a message $\mathrm{i}$ in time interval $T_{s} P_{\text {init }}$ is using as the lower bound of $P_{f}$ to ensure the delivery ratio and time delay while $p_{t h}$ is using as the upper bound of $P_{f}$ to control overhead

$R D_{i}\left(T_{s}\right)$ is calculated as follows: Let $M_{i}\left(T_{s}\right)$ be the total number of nodes with the message $i$ that the node encounters in $T_{s}$ seconds, and $N\left(T_{s}\right)$ be the total number of nodes that the node encounters in $T_{s}$ seconds. Then, the replications density for an arbitrary message is defined as

$$
R D_{i}\left(T_{s}\right)=\frac{M_{i}\left(T_{s}\right)}{N\left(T_{s}\right)}
$$

In the special case, when a node is isolated during the period, $T_{t h}$ seconds, the value of RD will be remain until a new node is encountered and the process of calculated the RD is started. When receiving a message, node checks if itself is the destination If not, computes MD to calculate the message forwarding probability and send message by this probability.

\subsection{Buffer Management Policy}

As mentioned above, when the network becomes congested, we consider dropping message with more hops and larger latency first. We use the information contained in the node itself to estimate the average latency and the average hop count of network. Then, we use the estimation values to calculate the priority weight of each message. Messages with more hops and larger latency correspond to smaller priority weights. When the network becomes congested, we drop the message with smallest priority weight first.

For node $i$ in the network, $m_{i}$ is the number of messages in its buffer, $T_{i j}$ is the living time of message $\mathrm{j}$ in node $i$ and $H_{i j}$. Is the hop count of message $j$ in node $i$. The estimation values and the priority weight are calculated as follows:

- The average latency estimation

$$
T_{i}=C_{1} * \frac{1}{m_{i}} \sum_{j=1}^{m_{i}} T_{i j}
$$

$C_{1}$ is the latency estimation constant, usually 1 4.

$$
\text { - } \quad \text { The average hop count estimation }
$$

$$
H_{i}=C_{2} * \frac{1}{m_{i}} \sum_{j=1}^{m_{i}} H_{i j}
$$

$C_{2}$ is the hop count estimation constant, usually $1 \sim 4$.

$$
\text { The priority weight function for node } i
$$

$$
W_{i}=\alpha * \exp \left(\frac{-T_{c l t}}{2 T_{i}}\right)+(1-\alpha) * \exp \left(\frac{-H_{c} C_{n i}}{H_{i}}\right)
$$

$\alpha$ is the latency term coefficient, $\alpha \in(0,1) . T_{c l t}$ is the current message living time. $H_{c}$ is the current message hop count. $C_{n i}$ is the estimation for the average number of neighbor nodes. The coefficients $C_{1}, C_{2}$ and $\alpha$ do affect the performance of NARP. These coefficients can be set to different values for different scenarios. Usually, we set $C_{1}=C_{2}=2.5$ and $\alpha=0.5$, if there is no special request

\subsection{Transmission Priority Policy}

When a new node is discovered, the two nodes will exchange a series of messages using the Vector Exchange Scheme [15]. Since the contact duration may not be enough to transmit all messages, both nodes priorities the messages to be transmitted according the forwarding probability message.

\section{SIMULATION AND RESULTS}

The ONE simulator [16] is used for simulation. The DTN network used for evaluation consists of six groups of nodes. Pedestrians (group 1 and group 3) can move anywhere in the map, but cars (group 2) can only move on roads. There are three groups of trams, group 4 is with broadcast interfaces, and group 5 and group 6 are normal. Most of the nodes in the

\begin{tabular}{|c|c|}
\hline Parameter & Value \\
\hline Node speed & $\begin{array}{l}\text { - Group 1: } 0.5 \sim 1.5 \mathrm{~m} / \mathrm{s}, 40 \\
\text { pedestrians } \\
\text { - Group 2: } 2.7 \mathrm{~m} / \mathrm{s} \sim 13.9 \mathrm{~m} / \mathrm{s}, 40 \\
\text { cars only on roads } \\
\text { - Group 3: } 0.5 \mathrm{~m} / \mathrm{s} \sim 1.5 \mathrm{~m} / \mathrm{s}, 40 \\
\text { pedestrians } \\
\text { - Group 4: } 7 \mathrm{~m} / \mathrm{s} \sim 10 \mathrm{~m} / \mathrm{s}, 2 \text { trams } \\
\text { with broadcast interfaces } \\
\text { - Group 5: } 7 \mathrm{~m} / \mathrm{s} \sim 10 \mathrm{~m} / \mathrm{s}, 2 \text { trams } \\
\text { - Group } 6: 7 \mathrm{~m} / \mathrm{s} \sim 10 \mathrm{~m} / \mathrm{s}, 2 \text { trams }\end{array}$ \\
\hline Transmission speed & $250 \mathrm{~KB} / \mathrm{s}(10 \mathrm{MB} / \mathrm{s}$ for group 4$)$ \\
\hline Transmission range & $10 \mathrm{~m}(1000 \mathrm{~m}$ for group 4$)$ \\
\hline Message size & $500 \mathrm{~KB} \sim 1 \mathrm{MB}$ \\
\hline Message TTL & 300min \\
\hline Number of nodes & 126 \\
\hline Scenario size & $4500 \mathrm{~m} \times 3400 \mathrm{~m}$ \\
\hline Simulation time & $12 \mathrm{~h}$ \\
\hline $\begin{array}{l}\text { Message creation } \\
\text { interval }\end{array}$ & One new message every 25 to $35 \mathrm{~s}$ \\
\hline
\end{tabular}
network are pedestrians and cars. There are ten rounds in the simulation. For group 1, group 2 and group 3, the node buffer size is $1 \mathrm{MB}$ for the first round and $2 \mathrm{MB}$ for the second round, and so on. For the groups of trams, the node buffer size is always 50MB. The simulation parameters are list in Table 1

Table 1. Simulation Parameters 


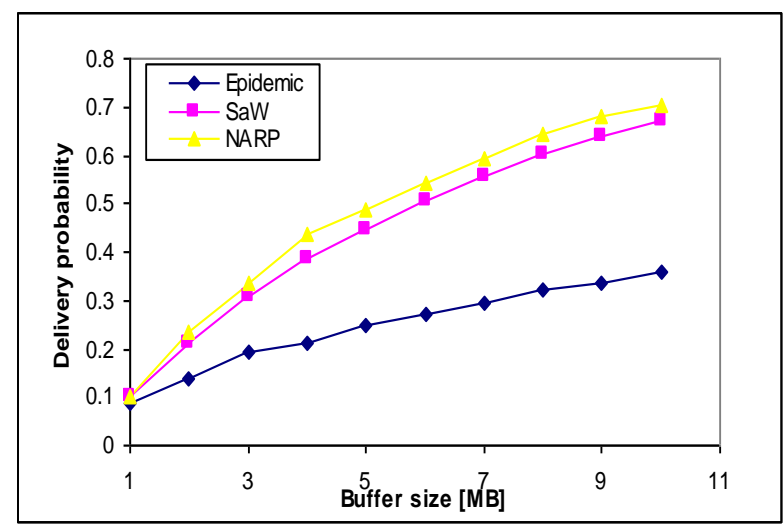

Fig 1: Delivery probability versus Buffer size.

Fig. 1 shows the delivery ratio as the buffer size is varied, for Spray and Wait Routing, Epidemic Routing, and NARP. The delivery ratios of all routing protocols increase as the buffer size increases. Among these DTN routing protocols, the NARP achieves the highest delivery ratio. This is because the NARP has better mechanism for message priority mechanism to determine their priority for forwarding and for dropping. However, as the buffer size increase the buffer constraint is removed and other protocols start to deliver more messages. Furthermore, for NARP routing, it can achieve a delivery ratio up to $83 \%$ higher than Epidemic routing and only $6.8 \%$ higher than Spray and Wait Routing.

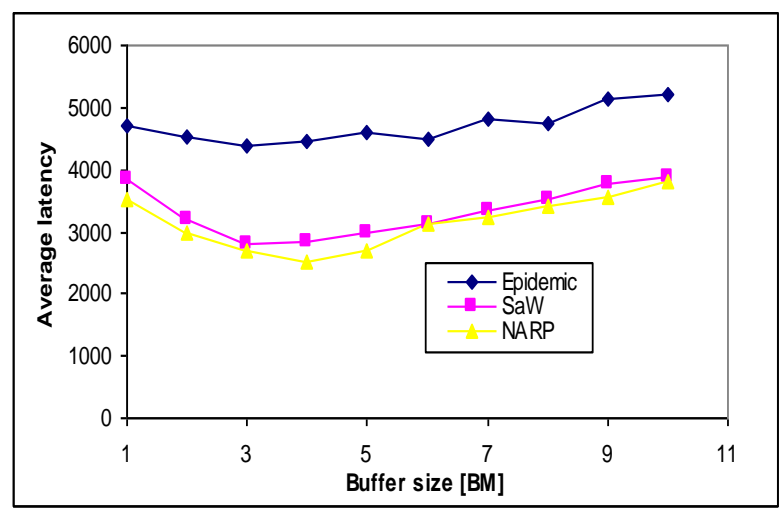

Fig 2: Average latency versus Buffer size.

Fig. 2 shows the average delay of a message as the buffer size varies. Similar to the delivery ratio, the result shows that the performance of NARP is better than those of Spray and Wait and Epidemic. The reason for the shorter average delay is because messages are prioritized and the network is not so congested as NARP uses an adaptive forwarding algorithm to minimize network congestion and messages are prioritized for forwarding by a node. The simulation results show that NARP achieves a $33.2 \%$ reduction in average delivery delay over Epidemic, and only 5.6\% reduction than Spray and Wait routing protocols.

Fig.3. NARP utilizes the ACK vector exchange mechanism to purge the redundant messages, as the simulation results indicate, the copies of each message are much less than Spray and Wait and Epidemic Routing protocols The simulation results show that NARP achieves a $86.6 \%$ reduction in average delivery delay over Epidemic, and only $7.8 \%$ reduction than Spray and Wait routing protocol.

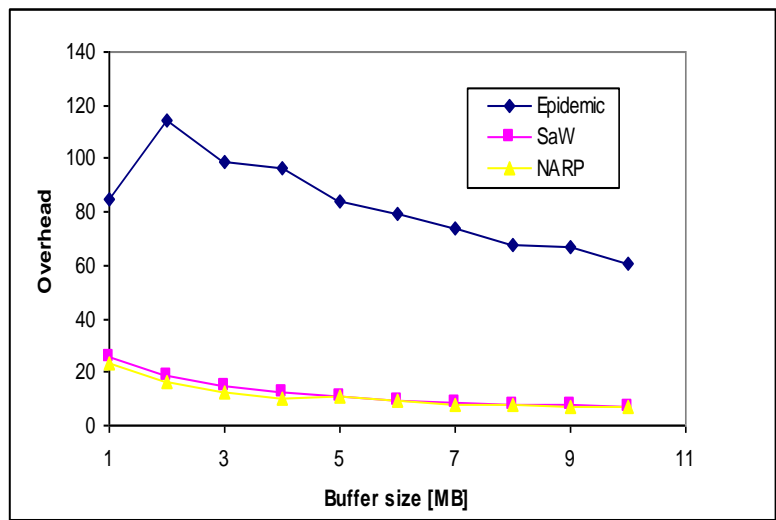

Fig 3: Overhead versus Buffer size.

\section{CONCLUSION}

Delay Tolerant Networks often use multi-copy routing schemes for message transmission. Multi-copy routing schemes, such as Epidemic Routing, usually lead to congestion problems. This paper presents a New Adaptive Routing Protocol (NARP) to solve congestion problems and improve the delivery ratio. The simulation results show that the NARP routing protocol significantly improves the delivery ratio and reduces the delivery delay and the network overhead.

\section{REFERENCES}

[1] P. Marshall. The disruption tolerant networking program, 2005.

http://www.darpa.mil/sto/solicitations/DTN/briefs.htm.

[2] P. Basu and T. Little, "Networked parking spaces: architecture and applications", Preceding of the 56th IEEE Vehicular Technology Conference (VTC 2002Fall), vol . 2, pp. 1153- 1157, 2002.

[3] S. Burleigh et al., "Delay-tolerant networking: An approach to interplanetary internet", in IEEE Communications Magazine, vol. 41, no. 7, pp. 128-136, June, 2003

[4] A. Doria, M. Udn, and D. P. Pandey, "Providing connectivity to the saami nomadic community", in Proc. 2nd Int. Conf. on Open Collaborative Design for Sustainable Innovation, Dec. 2002 .

[5] A. Vahdat and D. Becker, "Epidemic routing for partially connected ad hoc networks", Duke Tech Report CS2000-06, 2000

[6] A. Spyropoulos et al., "Spray and Wait: An Efficient Routing Scheme for Intermittently Connected Mobile Networks", ACM SIGCOMM Wksp. Delay Tolerant Networking, pp. 252- 259, August, 2005 .

[7] Spyropoulos T, Psounis K, Raghavendra C S., "SingleCopy Routing in Intermittently Connected Mobile Networks", in Sensor and Ad Hoc Communications and Networks, IEEE SECON 2004,pp. 235- 244, Oct., 2004

[8] A. Lindgren, A. Doria, and O. Scheln, "Probabilistic routing in intermittently connected networks", In SIGMOBILE Mobile Computing and Comm. Rev. vol. 7, no. 3, pp. 19- 20, 2003.

[9] J. Burgess et al., “ Maxprop: Routing for vehicle-based disruption tolerant networks", In Proc. IEEE Infocom, 
$25^{\text {th }}$ Int. Conf. on Computer Comm. pp. 1- 11, April, 2006 .

[10] Sushant Jain, Kevin Fall, and Rabin Patra, "Routing in a delay tolerant network", In ACM SIGCOMM Computer Comm. Rev, vol. 34, no. 4, pp. 145-158, October, 2004.

[11] Zhao W, Ammar M, Zegura E., "Controlling the Mobility of Multiple Data Transport Ferries in a DelayTolerant Network", In IEEE INFOCOM, pp. 1407 1418, .vol. 2, March, 2005

[12] Krifa A, C Barakat, Spyropoulos T., "An Optimal Joint Scheduling and Drop Policy for Delay Tolerant Networks", In World of Wireless, Mobile and Multimedia Networks (WoWMoM 2008 Int. Symposium), pp. :1 6, June, 2008.
[13] X. Zhang, G. Neglia, J. Kurose, and D. Twosley, "Performance modeling of epidemic routing," in Proceedings of Int. Federation for Information Processing (IFIP) Networking, pp. 535-546, 2006.

[14] Mamoun Hussein Mamoun, "Efficient DTN Routing Protocol", International Journal of Computer Applications, vol. 80, no. 9, pp. 16-19, October, 2013..

[15] A. Vahdat and D. Becker, "Epidemic routing for partially connected ad hoc networks", Technical Report CS 20006, Dept. of Computer Science, Duke University, Durham, NC 2000.

[16] Ari Keronen, and Jurg Ott, "The One simulator for DTN protocol evaluation", In SIMUTools, Rom, Italy, 2009. 